\title{
中国心理学前沿
}

\section{小学高年级儿童亲子依恋与欺负行 为的关系：同伴关系的中介作用}

\section{黄 茹王 柯 周守珍 *}

长江大学教育学院, 荆州, 434000

邮箱: hbeueb05hr@sina.com

摘 要: 目的: 探讨亲子依恋风格、同伴关系以及欺负行为在性别、年级等人 口学变量上的差异, 并进一步分析亲子依恋、同伴关系与欺负行为三者之间的关 系。方法: 采用亲子依恋量表、同伴关系问卷和 Olweus 儿童欺负问卷对 532 名 小学生高年级儿童进行问卷调查。结果: 亲子依恋的性别、年级在总分上差异显 著, 亲子依恋的性别在亲近倾向、信赖程度维度上也差异显著。欺负行为的性别、 年级在各个维度上差异显著, 男生在欺负行为的各个维度的得分显著地高于女 生。同伴关系在同伴接受维度上性别差异显著, 但无年级差异。亲子依恋两维度 分别与同伴关系中同伴接受维度显著正相关、同伴恐惧自卑维度显著负相关; 同 伴关系中的同伴接受维度和亲子依恋的两个维度与欺负行为的三个维度之间呈负 相关, 同伴关系中的同伴恐惧自卑维度与欺负行为的三个维度呈正相关。结论: 父母与孩子之间的亲子依恋水平能够显著地预测儿童在校的欺负行为; 同伴关系 在亲子依恋与欺负行为之间起着部分中介作用。 
关键词：小学高年级儿童；亲子依恋；同伴关系；欺负行为；中介作用

投稿日期：2019-04-15；录用日期：2019-05-20；发表日期：2019-06-30

\title{
The RelationshipBetween Parent-Child Attachment and Bullying Behavior in Senior Primary School Children:The Mediating Role of Peer Relationship
}

\author{
Huang Ru Wang Ke Zhou Shouzhen"
}

School of Education, Yangtze University, Jingzhou 434000

Abstract: Objective: To explore the differences of parent-child attachment style, peer relationship, bullying behavior in gender, grade and other demographic variables, and to further analyze the relationship among parent-child attachment, peer relationship and bullying behavior. Methods: the parent-child attachment scale, peer relationship questionnaire and Olweus child-bullying questionnaire were used to investigate 532 primary school children of senior grade. Results: the gender and grade of parent-child attachment had significant differences in total score, and the gender of parent-child attachment also had significant differences in intimacy tendency and trust degree. There are significant differences in gender and grade of bullying behaviors in all dimensions, and boys score significantly higher than girls in all dimensions of bullying behaviors. The gender difference in peer acceptance was significant, but there was no grade difference. The two dimensions of parent-child attachment are significantly positively correlated with the dimension of peer acceptance and the dimension of peer fear and inferiority, respectively. The two dimensions of peer acceptance and 
parent-child attachment in peer relationship are negatively correlated with the three dimensions of bullying behavior while the three dimensions of peer fear and inferiority in peer relationship are positively correlated with the three dimensions of bullying behavior. Conclusion: the level of parent-child attachment between parents and children can significantly predict bullying behaviors in school. Peer relationship plays a part of mediating role between parent-child attachment and bullying behavior. Key words: Senior primary school children; Parent-child attachment; Peer relations; Bullying; Being bullied

Received: 2019-04-15; Accepted: 2019-05-20; Published: 2019-06-30

Copyright @ 2019 by author(s) and SciScan Publishing Limited.

This work is licensed under a Creative Commons Attribution 4.0 International License. https://creativecommons.org/licenses/by/4.0/

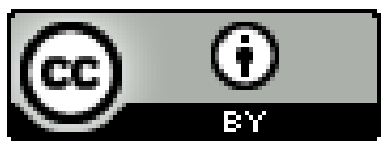

\section{1 引言}

近年来，中小学关于校园欺负的事件屡见不鲜，甚至随着年级的递升，这 一现象呈现愈演愈烈的趋势, 校园欺负也因此受到人们的关注。最早专门研究 欺负行为的挪威心理学家 Olweus 认为，欺负是攻击的一个子集，是指力量强大 的儿童经常利用言语或身体方式给弱小儿童带来伤害性行为 $[1]$ 。欺凌事件在 各个国家和地域都曾发生, 校园更是欺凌多发的地点。很多研究表明, 儿童的 攻击（欺凌）倾向与父母与孩子的亲近程度成反比。例如，与父母亲近程度低 的儿童较易发生攻击行为，导致在校园内难以获得良好的同伴关系 [2]。此外， 同伴关系更是直接影响到学生在校园内的生活质量。有研究发现受欺负者难以 被同伴接纳甚至会被拒绝；同时，较差的接纳关系又会导致儿童的欺负行为， 从而形成一系列恶性循环事件 [3]。由此可见，欺负行为的产生与同伴关系、 
亲子依恋有着密不可分的联系。

最早系统地探究依恋问题的是英国的精神分析学家 Bowlby，他认为依恋可以 定义为个体与特定的他人形成牢固的情感纽带的倾向。陈丽君对亲子依恋有了进 一步阐述，即为婴孩与第一照看人 (一般是母亲) 之间建立起来的情感联结状态 [4]。拥有安全依恋的儿童有更多的积极行为，在同伴交往中拥有更多的人际吸 引力，很少出现消极和攻击行为 [5]。同伴关系是一种同龄人或心理发展水平相 当的个体间在交往过程中建立和发展起来的人际关系 [6]。皮亚杰做了同伴关系 对儿童及青少年发展的影响作用最早的研究。根据皮亚杰的观点，形式运算阶段 不仅是青少年生理发展的高速阶段，更是其心理和社会性发展的高速阶段，亲子 关系、同伴关系与青少年的社会化有着密切的联系。岳永华等人 [7]的研究发现 亲子依恋较差的个体往往会出现较多的问题行为，陈武、李董平等人 $[8]$ 发现在 不良的亲子依恋环境下生长的青少年更容易结交越轨同伴。李锦萍 [9]研究发现 亲子依恋质量越差，个体出现攻击行为的可能性也越高，缺乏安全与信任感的个 体在人际互动中充满敌意认知，会消极应对外界环境，在学校更难拥有良好的同 伴关系。目前，国内鲜有将同伴关系视为亲子依恋与欺负行为的中介因子的研究。 故本研究旨在探究同伴关系在亲子依恋与欺负行为之间的中介效应, 以期为家长 和教师提供儿童教育的实证研究依据，达到降低校园欺负行为发生概率的目标。

\section{2 研究方法}

\section{1 研究对象}

以小学高年级学生为研究对象，采用分层整群抽样抽取被试。共回收 532 份有效问卷，分别为四年级 167 人，五年级学生 150 人，六年级 131 人; 男生 248 人，女生 200 人。

\section{2 研究工具}

\subsection{1 小学生亲子依恋量表}

小学生亲子依恋量表是依据于海琴修订的儿童亲子依恋量表制定，该量表 
分为两个维度, 亲近倾向 (指对自己想得到父母帮助的自我知觉) 和信赖程度 (指 儿童对父母作为支持源可靠性的认识）。量表共 15 题，该问卷分为正向计分和 反向计分，1、6、9、14 题采用反向计分，其他题为正向计分。该问卷得分越高， 说明依恋安全水平越高。该量表的内部一致性系数分别为 0.84 (父亲) 和 0.81 (母 亲），具有良好的信效度。

\subsubsection{Olweus 儿童欺负问卷}

采用张文新修订的 Olweus 儿童欺负问卷中的欺负与受欺负两个分量表对儿 童欺负情况进行统计。量表分为直接言语欺负、关系欺负和身体欺负 3 个维度, 整个量表共有 12 个项目。量表采用五级评分。两个分量表都有较好的内部一致 性（欺负：0.72, 受欺负：0.78）。

\subsection{3 同伴关系问卷}

使用的是邹泓修订的同伴关系问卷对同伴关系进行调查，该问卷考察了儿童在 与他人相处过程中的自我感觉。问卷分为同伴接受和同伴恐惧自卑 2 个维度, 共 30 个项目，1 到 20 题是同伴接受分量表，21 到 30 题是同伴恐惧自卑分量表，其中同 伴接受分量表除 $1 、 3 、 7 、 11 、 17$ 项目外其余项目要反向计分，同伴恐惧自卑分量 表项目都采用正向计分。量表采用四级计分。该问卷的内部一致性系数是 0.91 。

\section{3 数据处理}

使用 SPSS 21.0 统计分析软件对回收的数据进行统计分析，主要采用描述统 计、 $t$ 检验、相关分析、方差分析和回归分析等方法。

\section{3 结果}

\section{1 亲子依恋、同伴关系和欺负行为的性别、年级差异}

如表 1 所示，性别在亲子依恋的各个维度上存在显著差异（ $p<0.05 ）$ ，女 生在亲子依恋的各个维度上的得分显著高于男生，小学高年级女生与父母的关系 更加亲密。如表 2 所示, 年级在亲子依恋总分上存在显著差异 $(F=3.096, p<0.05)$, 通过事后检验发现, 五年级儿童的亲子依恋程度最高, 四年级其次, 六年级最低, 
在亲近倾向和信赖程度上无统计学意义。由表 3 可知，性别在欺负行为各维度 上存在显著差异（ $p<0.001 ）$ ，男生在各维度上得分均高于女生。由表 4 可知， 年级在欺负行为三个维度上存在显著差异 $(p<0.001)$ ，通过事后检验发现， 四年级学生在欺负行为各维度上得分高于五年级，五年级得分高于六年级。由 表 5 可知性别在同伴接受水平上差异显著 $(p<0.01)$ ，女生同伴接受显著高于 男生，而在同伴恐惧自卑水平上无显著性差异。

表 1 亲子依恋及各维度在性别上的差异检验 $(M \pm S D)$

Table 1 Parent-child attachment and gender differences in each dimension( $M \pm S D$ )

\begin{tabular}{cccc}
\hline & \multicolumn{2}{c}{ 性别 } & \multirow{2}{*}{$t$} \\
\cline { 2 - 3 } & 男 & 女 & $-2.534^{*}$ \\
亲近倾向 & $19.06 \pm 4.376$ & $20.14 \pm 4.564$ & $-2.441^{*}$ \\
信赖程度 & $24.67 \pm 4.601$ & $25.74 \pm 4.545$ & $-2.759^{* * * *}$ \\
亲子依恋 & $43.73 \pm 7.950$ & $45.87 \pm 8.382$ & \\
\hline
\end{tabular}

注: $* p<0.05, * * p<0.01, * * * p<0.001$, 下同。

表 2 亲子依恋在年级上的差异检验 $(M \pm S D)$

Table 2 Parental attachment test at the grade level $(M \pm S D)$

\begin{tabular}{cccccc}
\hline & 1)四年级 & 2)五年级 & (3)六年级 & $F$ & $L S D$ \\
\hline 亲近倾向 & $19.75 \pm 4.821$ & $19.97 \pm 4.250$ & $18.77 \pm 4.242$ & 2.836 & (2) $>$ (1) > (3) \\
信赖程度 & $25.41 \pm 4.692$ & $25.48 \pm 4.338$ & $24.44 \pm 4.733$ & 2.240 & \\
亲子依恋 & $45.16 \pm 8.653$ & $45.45 \pm 7.442$ & $43.21 \pm 8,317$ & $3.096^{*}$ & \\
\hline
\end{tabular}

表 3 欺负行为各维度在性别上的差异检验 $(M \pm S D)$

Table 3 Gender differences in the dimensions of bullying behavior $(M \pm S D)$

\begin{tabular}{cccc}
\hline & \multicolumn{2}{c}{ 性别 } & \multirow{2}{*}{$t$} \\
\cline { 2 - 4 } & 男 & 女 & $5.656^{* * *}$ \\
\hline 直接言语欺负 & $3.10 \pm 3.397$ & $1.56 \pm 2.051$ & $3.162^{* * *}$ \\
关系欺负 & $2.11 \pm 3.001$ & $1.32 \pm 2.114$ & $5.227^{* * *}$ \\
直接身体欺负 & $2.42 \pm 3.124$ & $1.12 \pm 1.827$ & \\
\hline
\end{tabular}

表 4 欺负行为各维度在年级的差异检验 $(M \pm S D)$

Table 4 Difference test of bullying behavior in different grades $(M \pm S D)$

\begin{tabular}{cccccc}
\hline & 1)四年级 & (2)五年级 & (3)六年级 & $F$ & $L S D$ \\
\hline 直接言语欺负 & $3.05 \pm 3.409$ & $2.22 \pm 2.768$ & $1.82 \pm 2.420$ & $6.935^{* * *}$ & (1) $>$ (2) $>$ (3) \\
关系欺负 & $2.39 \pm 3.175$ & $1.41 \pm 2.280$ & $1.34 \pm 2.197$ & $7.792^{* * *}$ & (1) $>$ (2) (3) \\
直接身体欺负 & $2.47 \pm 3.217$ & $1.51 \pm 2.407$ & $1.4 \pm 2.10$ & $7.663^{* * *}$ & (1)>(2)>(3) \\
\hline
\end{tabular}


表 5 同伴关系各维度在性别上的差异检验 $(M \pm S D)$

Table 5 Gender differences in the dimensions of peer relationships $(M \pm S D)$

\begin{tabular}{cccc}
\hline & \multicolumn{2}{c}{ 性别 } & \multirow{2}{*}{$t$} \\
\cline { 2 - 3 } & 男 & 女 & $-2.970^{* *}$ \\
同伴接受 & $60.91 \pm 8.576$ & $63.33 \pm 8.592$ & 0.735 \\
同伴恐惧自卑 & $19.80 \pm 6.416$ & $19.60 \pm 6.224$ & \\
\hline
\end{tabular}

\section{2 同伴关系、亲子依恋、欺负行为的相关关系}

小学高年级儿童同伴关系、亲子依恋与欺负行为的相关关系研究表明，亲 子依恋的两个维度与同伴关系中的同伴接受存在显著正相关, 亲子依恋的两个维 度与同伴关系中的同伴恐惧自卑存在显著负相关，同伴关系中的同伴接受与欺 负行为的三个维度呈负相关，同伴关系中的同伴恐惧自卑与欺负行为的三个维 度呈现显著正相关，亲子依恋的两个维度与欺负行为的三个维度均呈现显著负 相关, 具体见表 6 。

\section{表 6 同伴关系、亲子依恋与欺负行为的相关分析}

Table 6 Correlation Analysis of Peer Relationship, Parent-Child Attachment and Bullying Behavior

\begin{tabular}{lccccccc}
\hline & 1 & 2 & 3 & 4 & 5 & 6 & 7 \\
\hline 1. 亲近倾向 & 1 & - & - & - & - & - & - \\
2. 信赖程度 & $0.630^{* *}$ & 1 & - & - & - & - & - \\
3. 同伴接受 & $0.345^{* *}$ & $0.379^{* *}$ & 1 & - & - & - & - \\
4. 同伴恐惧自卑 & $-0.280^{* *}$ & $-0.315^{* *}$ & $-0.525^{* *}$ & 1 & - & - & - \\
5. 直接言语欺负 & $-0.209^{* *}$ & $-0.182^{* *}$ & $-0.210^{* *}$ & $0.198^{* *}$ & 1 & - & - \\
6. 关系欺负 & $-0.241^{* *}$ & $-0.213^{* *}$ & $-0.292^{* *}$ & $0.304^{* *}$ & $0.645^{* *}$ & 1 & - \\
7. 直接身体欺负 & $-0.167^{* * *}$ & $-0.173^{* *}$ & $-0.244^{* *}$ & $0.257^{* *}$ & $0.645^{* *}$ & $0.706^{* *}$ & 1 \\
\hline
\end{tabular}

\section{3 同伴关系在亲子依恋与欺负行为两者之间的中介效应}

通过路径分析来检验小学高年级儿童亲子依恋、同伴关系对欺负行为的影 响。将亲子依恋作为自变量, 同伴关系的两维度作为因变量来进行回归分析, 在同伴接受对亲子依恋的回归模型中, $F$ 值为 $85.810(p<0.001)$, 满足建立 线性模型的条件, 其中调整 $R^{2}$ 为 0.159 , 说明亲子依恋 $15.9 \%$ 的变异量可以被 同伴接受解释, 模型拟合良好。在交往恐惧自卑对亲子依恋的回归模型中, $F$ 值 
为 $54.415(p<0.001)$, 表明线性关系显著，能够建立线性模型，本模型的调 整 $R^{2}$ 为 0.107 , 说明交往恐惧自卑这一变量可以解释亲子依恋 $10.7 \%$ 的变异量, 拟合优度良好。再将亲子依恋作为自变量, 将欺负行为三个变量作为因变量来进 行回归分析。在亲子依恋对欺负行为的回归模型中, $F$ 值分别为 $21.955(p<0.001)$ 、 $30.134 （ p<0.001) 、 16.372 （ p<0.001)$ ，满足建立线性模型的条件，其中 调整 $R^{2}$ 分别为 $0.045 、 0.061 、 0.033$, 模型拟合良好。将同伴关系作为自变量, 将欺负行为作为因变量进行回归分析。探究了同伴关系对欺负行为的预测效应, 在回归模型中， $F$ 值为 $12.943(p<0.001)$ ，表明线性关系显著，满足了条件。 该模型的调整 $R^{2}$ 为 0.051 , 说明同伴关系两个维度对直接言语欺负的预测力达 到了 $5.1 \%$, 拟合度良好。在同伴关系各维度对关系欺负的回归模型中, $F$ 值为 $29.358 （ p 0.001 ） ，$ 表明线性关系显著，该模型的调整 $R^{2}$ 为 0.113 ，说明同伴关 系各个维度对关系欺负的预测力达到了 $11.3 \%$ ，拟合度良好。在同伴关系对直接 身体欺负的回归模型中， $F$ 值为 $20.005(p<0.001)$ ，线性关系显著，该模型 的调整 $R^{2}$ 为 0.078 , 说明同伴关系各个维度对直接身体欺负的预测力达到了 $7.8 \%$, 拟合度良好。

通过前面的回归分析，我们得出儿童的亲子依恋可以显著预测儿童的同伴 关系，而儿童的同伴关系可以显著预测儿童的欺负行为，儿童的亲子依恋对欺 负行为也存在着显著的预测作用。当我们把同伴关系的两维度纳人到亲子依恋 与欺负行为的模型中后，如表 7 所示。

表 7 亲子依恋、同伴关系和欺负行为的回归分析表

Table 7 Regression analysis table of parent-child attachment, peer relationship and bullying behavior

\begin{tabular}{|c|c|c|c|c|c|}
\hline 自变量 & 因变量 & $R^{2}$ & $F$ & $\bar{\beta}$ & $t$ \\
\hline $\begin{array}{c}\text { 亲子依恋 } \\
\text { 同伴接受 } \\
\text { 同伴恐惧自卑 }\end{array}$ & 直接言语欺负 & 0.072 & $11.459 * * *$ & $\begin{array}{c}-0.144 \\
-0.101 \\
0.098 \\
\end{array}$ & $\begin{array}{c}-2.842 * * \\
-0.811 \\
1.800\end{array}$ \\
\hline $\begin{array}{c}\text { 亲子依恋 } \\
\text { 同伴接受 } \\
\text { 同伴恐惧自卑 }\end{array}$ & 关系欺负 & 0.131 & $22.354 * * *$ & $\begin{array}{c}-0.134 \\
-0.141 \\
0.186\end{array}$ & $\begin{array}{c}-2.737 * * \\
-2.592 * \\
3.540 * * *\end{array}$ \\
\hline
\end{tabular}


续表

\begin{tabular}{cccccc}
\hline 自变量 & 因变量 & $R^{2}$ & $F$ & $\beta$ & $t$ \\
& & & & & \\
亲子依恋 & & & & -0.084 & -1.683 \\
直接身体欺负 & 0.088 & $14.336^{* * *}$ & -0.124 & $-2.225^{*}$ \\
同伴恐惧自卑 & & & & 0.165 & $3.056^{* *}$ \\
\hline
\end{tabular}

根据 Baron 和 Kenny 的检验方法，本研究中，亲子依恋对同伴接受、同伴 恐惧自卑存在较强的影响力（ $\beta=0.402,0.330)$; 亲子依恋对直接言语欺负、 关系欺负和直接身体欺负存在影响 $(\beta=0.217,0.252,0.188)$ 同伴关系的两个 维度对欺负行为的三个维度存在影响 $(\beta=0.147,0.121,0.183,0.208,0.150$, 0.178 ）。当把同伴关系引人亲子依恋和欺负行为的回归方程中, 亲子依恋对直 接言语欺负、关系欺负和直接身体欺负的 $\beta$ 值都减少了 $(\beta=0.144,0.134,0.084)$ （ $\beta$ 的绝对值反映了自变量对因变量的影响程度），与未引人同伴关系时亲子 依恋对欺负行为的影响力减弱了，以上都满足 Baron 和 Kenny 的主张确定中介变 量需要满足的条件, 因此可以认为同伴关系是亲子依恋和欺负行为的中介变量, 起着部分中介作用。

\section{4 讨论}

\section{1 人口学变量对各研究变量的差异分析}

从性别来看，男生与父母的亲近倾向和信赖程度显著低于女生。这一结论 与李凌 $[10$ 的研究结论一致。研究显示, 女孩与母亲相关的依恋分数比男孩的高, 可能是社会对男孩提出更多独立、坚强的要求，导致男孩对父母的依恋少于女生。 从年级来看，六年级儿童的亲子依恋得分明显低于四五年级，与于海琴和周宗 奎 $[11]$ 得出的结论相一致。可能是由于随着儿童年龄的增长，六年级儿童逐 渐进人青春期，叛逆期让孩子与父母的依恋质量下降。

从性别上来看，男生和女生在欺负行为问卷的三个维度上具有极其显著的 差异, 女生在欺负行为的三个维度上的得分都显著地低于男生。这一结论与雷 雾和张雷等人 $[12]$ 的研究相一致, 男女在欺负行为上存在显著差异, 男生比 女生受到更多的欺负。通过方差分析得出, 四年级的欺负行为各项得分高于五 
年级, 五年级各项得分高于六年级, 张文新的研究也给出相近的结论 $[13]$ 。 这可能是由于六年级儿童面临着升学的压力, 更多精力付出在学习上, 同学之 间的摩擦减少。

从同伴关系问卷中的同伴接受维度来看，男女生之间存在显著差异，女生 的同伴接受程度远高于男生，男女生在同伴恐惧自卑维度上无显著差异。由此 可知，女生的同伴关系要显著好于男生。这一结论也与张戎骏 [14]在同伴关 系中的研究结论相一致。通过综合文献和其他研究数据的结果分析，同伴关系 之所以会出现这样的性别差异，原因有如下几方面：第一，在解决冲突方面, 一般女生相对于男生更倾向于采用温和的方式。第二, 可能受到社会文化的影响, 女生会被教育要温和柔顺，而男生一般被教育要刚强勇敢。第三，男女生在情 绪的表达上存在显著差异。

\section{2 小学高年级儿童同伴关系、亲子依恋与欺负行为的相关} 研究

亲子依恋、同伴关系和欺负行为三者之间存在显著相关（见表 6 ）。亲子依 恋的两个维度与欺负行为的三个维度存在显著的负相关，儿童亲子依恋的水平 越高, 儿童受欺负和欺负行为发生率越低。这一结论与陈健芷等学者的研究结 论相一致。依恋关系能够在一定程度上预测个人欺凌的程度 $[15]$ 。说明儿童 在家庭中形成安全的依恋有助于减少其在学校被欺负的几率。

同伴关系两个维度和欺负行为三个维度之间也存在显著相关。同伴接受程 度越高，欺负行为发生几率越低，同伴恐惧自卑程度越高，欺负行为发生几率 越高。这一结论与前人的研究结果一致。欺负行为会因为同伴关系的不同而表 现出显著的差异 $[16]$ 。学生拥有的朋友数与其受欺负发生的频率呈负相关 $[17$ 。 儿童的朋友数量越多，在同伴交往中可能会获得更多的帮助和支持，在群体中 朋友的支持会减少受欺负事件的发生。因此在儿童的交往中，同伴关系的建立 至关重要, 而要拥有建立良好人际关系的能力需要家庭和学校的共同努力。

亲子依恋两维度与同伴关系两维度之间也存在着显著相关。儿童亲近倾向 和信赖程度越高，同伴接受程度也就越高；同时，儿童的亲近倾向和信赖程度 
越高, 同伴恐惧自卑程度就越低。依恋程度比较好的儿童能够与他人更好地相处, 对于新鲜事物多报以积极的态度，他们的攻击性相比于其他儿童会更低，因此 这些儿童会拥有更好的同伴关系，而不良亲子依恋的儿童与这些亲子依恋良好 的儿童恰恰相反, 所以同伴关系较差 [18]。因此, 亲子依恋对儿童的同伴关 系有着重要的影响。

\section{3 同伴关系在亲子依恋与欺负行为两者之间的中介效应}

从研究结果可以看出, 小学高年级儿童亲子依恋与欺负行为之间存在显著 相关，小学高年级儿童的亲子依恋可以显著预测欺负行为。亲子依恋、同伴关 系和欺负行为三者之间均存在显著相关。但是在亲子依恋与欺负行为中引人同 伴关系后，亲子依恋对欺负行为的影响变弱了，说明亲子依恋可能通过同伴关 系的中介作用来影响欺负行为，本研究也通过回归分析验证了这一结论。按照 Baron 和 Kenny 确定中介变量的四个条件, 充分说明了同伴关系的部分中介作用, 即小学高年级儿童的亲子依恋会通过同伴关系间接影响欺负行为。生态系统理 论认为, 家庭是个体成长过程中最重要的微观系统 [19]，而学校是除家庭以 外对其影响最大的微系统。对于儿童成长来说，家庭中良好亲子依恋关系的形 成和学校内良好同伴关系的建立十分重要。所以家长在儿童成长早期要注重亲 子关系的培养, 教师要注重引导儿童学习人际交往方面的知识, 提高儿童同伴 关系水平。

综上所述，儿童与父母的亲子依恋是否良好直接影响到儿童在校园内的同 伴关系状况，而同伴关系会直接影响儿童在学校是否会被欺负，因此我们可以 通过改善儿童的亲子依恋状况和同伴关系状况干预儿童在学校的欺负与受欺负 行为。

\section{参考文献}

[1] Olweus D, Alsaker F D.The negative shoa-term and long-term effects of victimization by peers $[\mathrm{R}]$.Paper presented at the 60th biennial conference for the Society for Research on Child Development Seatde, 1993. 
$[2]$ 孟仙, 余毅震, 刘卓雅, 等.小学高年级儿童攻击行为与亲子依恋关系 $[\mathrm{J}]$. 中国学校卫生，2011，32（8）：901-903.

[3] 肖少北, 刘丽琼, 朱铭, 等.欺负及受欺负小学生同伴关系分析 [ $\mathrm{J}]$.中 国学校卫生，2011，32（11）：1314-1315+1318.

[4] 陈丽君. 青少年依恋的发展特点及其与学校适应的相关研究 [D ]. 福建师 范大学, 2005 .

[5] Shaffer D R.SociallPer sonality Development [ M ]. California: Brooks Cole Publishing Company, 1994: 177-208.

[6]邹泓.同伴关系的发展功能及影因素 [ J ] . 心理发展与教育, 1998.

[7] 岳永华, 张光珍, 陈会昌. 儿童的依恋类型与其问题行为的关系 $[\mathrm{J}]$. 心理科学, $2010(2): 318-320$.

[8] 陈武，李董平，鲍振宙，等.亲子依恋与青少年的问题性网络使用：一个 有调节的中介模型 $[\mathrm{J}$ ] . 心理学报，2015，47（5）：611-623.

[9] 李锦萍, 李董平, 张卫. 亲子依恋、学校联结对初中生攻击性行为的影响 $[\mathrm{J}]$. 中国健康心理学杂志，2016，24（1）：68-72.

[10］于海琴，周宗奎.小学高年级儿童亲子依恋的发展及其与同伴交往的关系 [ J ] . 心理发展与教育，2002（4）：36-40.

[11 ] 李凌.早期养育经验与母子依恋水平的相关研究 [D ] . 心理科学, 2005, $28(3): 699-701$.

[12]雷雾，张雷.初中生受欺负状况的某些预测变量 $[\mathrm{J}$ ] . 心理学探新，2002 ( 4 ) : 38-43. https://doi.org/10.1111/an.2002.43.5.38.2

[13] 张文新. 中小学生欺负 / 受欺负的普遍性与基本特点 $[\mathrm{J}]$. 心理学报, 2002 ( 4 ) : 387-394. https://doi.org/10.1023/A:1021641222519

[14］张戎骏.初中生同伴关系现状与应对一一成都市 7 所初中为例 $[\mathrm{D}]$. 四川师范大学，2015.

[ 15$]$ 陈世平, 乐国安. 关于学校中儿童欺负行为的研究 $[\mathrm{J}]$. 广州大学学报 (社 会科学版)，2006（1）：42-46.

[16]蔡春凤. 小学儿童的心理行为特征、同伴接纳、拒绝与受欺负的关系研究 
[D ］. 华中师范大学，2006.

[17] 俞国良, 辛自强, 罗晓路.学习不良儿童孤独感、同伴接受性的特点及其 与家庭功能的关系 $[\mathrm{J}$ ] . 心理学报，2000（1）：59-64.

[18］陈健芷，刘昭阳，刘勇.初中生受欺负现状及其与亲子依恋和同伴关系的 关系 [ J ] . 中国临床心理学杂志，2013，21（5 ）：795-799.

[19] Bronfenbrenner U.Making human beings human: Bio-ecological perspectives on human development [ M ] .Thousand Oaks, CA: Sage, 2005. 